\title{
Cystic fibrosis: addressing the transition from pediatric to adult-oriented health care
}

This article was published in the following Dove Press journal:

Patient Preference and Adherence

10 December 2013

Number of times this article has been viewed

\author{
James L Kreindler ${ }^{1,2}$ \\ Victoria A Miller ${ }^{1,3}$ \\ 'The Children's Hospital of \\ Philadelphia, ${ }^{2}$ Department of \\ Pediatrics, ${ }^{3}$ Department of \\ Anesthesiology and Critical Care \\ Medicine, Perelman School of \\ Medicine at the University of \\ Pennsylvania, Philadelphia, PA, USA
}

Correspondence: James L Kreindler Children's Hospital of Philadelphia, 34th and Civic Center Boulevard, Philadelphia, PA 19104, USA Tel +I 2I5 5903749 Email kreindler@email.chop.edu

\begin{abstract}
Survival for patients with cystic fibrosis (CF) increased to nearly 40 years in 2012 from the early childhood years in the 1940s. Therefore, patients are living long enough to require transition from pediatric CF centers to adult CF centers. The goal of transition is for the young adult to be engaged in the adult health care system in ways that optimize health, maximize potential, and increase quality of life. A successful transition promotes autonomy and responsibility with respect to one's own health. Currently, there is an information gap in the literature with respect to psychological models that can help guide informed transition processes. In this review, we establish the framework in which transition exists in CF; we review some of the published literature from the last 20 years of experience with transition in CF centers around the world; and we discuss psychological models of pediatric illness that can help to explain the current state of transition to adult-oriented care from pediatric-oriented care and help to formulate new models of ascertaining readiness for transition. Finally, we look at our current knowledge gaps and opportunities for future research endeavors.
\end{abstract}

Keywords: cystic fibrosis, transition, adolescent, social-ecological model of AYA readiness for transition, SMART

\section{Introduction}

Cystic fibrosis (CF) is often defined in one form or another as the most common severely life-shortening genetic disease in Caucasians. There is a smaller, but significant, incidence of CF in African American, Hispanic, and Asian peoples. CF is caused by abnormalities in the expression and/or function of the cystic fibrosis transmembrane conductance regulator, that result in altered salt and water transport across epithelial surfaces in the gastrointestinal and respiratory tracts, the reproductive system, and the sweat glands. Abnormal salt and water transport in the lungs and gastrointestinal tract cause the majority of significant morbidity and mortality in patients with CF. Fortunately, advances in care and novel therapies have led to an extraordinary increase in survival for CF patients to nearly 40 years in 2012 from early childhood years in the 1940s. Therefore, patients are living long enough to require transition from pediatric CF centers to adult $\mathrm{CF}$ centers. The goal of transition is for the young adult to be engaged in the adult health care system in ways that optimize health, maximize potential, and increase quality of life. ${ }^{1}$ In addition, a successful transition should promote autonomy and responsibility with respect to one's own health. ${ }^{1}$

There are numerous reviews that address the current state of affairs in transition of a CF patient's care from a pediatric CF center to an adult CF center. Additionally, there are multiple opinion pieces and anecdotal reports that highlight the complexities 
and challenges of navigating this transition with our patients. Nonetheless, there is an information gap in the literature with respect to psychological models that can help guide informed transition processes. In this review, we review the rationale for transition in CF in order to establish the framework in which this transition exists. We then review some of the published literature from the last 20 years of experience with transition in CF centers around the world. Following this review, we discuss psychological models of pediatric illness that can help to explain the current state of transition to adult-oriented care from pediatric-oriented care and help to formulate new models of ascertaining readiness for transition. Finally, we look at our current knowledge gaps and opportunities for future research endeavors.

\section{Rationale for transition in CF}

$\mathrm{CF}$ is a multisystem disease that bears a heavy treatment burden. The vast majority of $\mathrm{CF}$ patients $(>90 \%)$ receive supplemental, exogenous, pancreatic enzymes to treat loss of pancreatic exocrine tissue. Such pancreatic insufficiency also necessitates daily supplementation of fat-soluble vitamins. Survival of infancy and early childhood because of better nutrition from the use of pancreatic enzyme replacement meant more children with CF succumbing to respiratory failure later in life. Respiratory failure arises secondary to a vicious cycle of mucus retention, infection, inflammation, and tissue damage, and remains the most common cause of mortality in CF. Therefore, there are many therapies designed to treat each part of the cycle.

Medications designed specifically to improve mucus clearance for patients with CF include inhaled mucolytic and osmotic agents that may take 15-20 minutes to complete. These medications are most often used in combination with some form of chest physiotherapy that consists of percussive forces applied to the chest wall for approximately 20-30 minutes in order to loosen mucus from the airway walls. Percussive therapy may take the form of manual chest percussion or use of a mechanical device such as a percussive vest. This therapy, in turn, is done in combination with specific breathing techniques, such as active cycles of breathing and huff coughing. Exercise may also be used as a physiotherapy strategy, and the combination of standard therapy and exercise improves lung function above that of standard therapy alone. ${ }^{2}$ As patients with CF age, they are often asked to take regular anti-inflammatory medications and inhaled antibiotics, further adding to a lengthy and complex regimen. Also, as patients age, disease complexity may increase with the presence of CF-related diabetes and CF-related liver disease, each of which may require additional therapies. Most recently, a new cystic fibrosis transmembrane conductance regulator-specific approach to therapy has become a reality. ${ }^{3}$

All told, patients with CF may spend an hour or many hours per day dedicated to therapies, ${ }^{4}$ and this complexity clearly increases over time. ${ }^{5}$ When CF patients are young, their parents perform the majority of this care. As children develop and mature, they are expected to participate more actively in their care, eventually assuming total responsibility. However, there is variability in the way in which families perceive, negotiate, and manage the transition to greater independence for self-management tasks, and decreases in adherence during adolescence are common. ${ }^{6,7}$ In addition, while parents are likely to remain an important source of support and guidance for youth with CF well into young adulthood, ${ }^{8}$ parental over-involvement may send the message that the adolescent is incompetent to manage the illness on his/her own, disrupt effective problem-solving, and increase parent-youth conflict. ${ }^{9}$ Therefore, the way in which a family manages the transition to greater independence during childhood and early adolescence is likely to have a strong impact on perceptions of transition, patient readiness, and outcomes of transition. It is important for models of transition to adult care and related interventions/programs for patients with $\mathrm{CF}$ to: consider transition as a process that begins early in the patient's development; engage both the patient and the parents in the transition process, recognizing that parents are likely to remain involved after the actual transfer takes place; and appreciate each family's individual circumstances and the broader context of transition, including the presence of other social supports and financial/insurance arrangements.

\section{Models of care transition}

The need for transition from pediatric care to adult care carries a number of implications. First and foremost, it implies that the child who is transitioning is dealing with a chronic illness. Therefore, the process of transition should be guided by the same principles that drive chronic care, ie, appreciation for the developmental stage of the patient, recognition of the dynamic nature of relationships between the patient, the patient's family and community, and the health care team, and sensitivity to cultural norms. ${ }^{10}$ Here, we discuss several conceptual models that can be used to guide our understanding of the key features of the transition to adult care. First, we review the published literature relating to transition of care in $\mathrm{CF}$, which has primarily relied on the concept of patient readiness. Second, we review two 
conceptual models of adjustment to pediatric chronic illness, ie, the pediatric psychosocial preventative health model and the medical traumatic stress model, with particular attention to the implications for transition. Third, we review a model of readiness for transition called SMART (Social-Ecological Model of AYA Readiness for Transition $)^{10}$ that draws on the aforementioned models of adjustment to chronic pediatric illness, as well as Bronfenbrenner's social-ecological model of development. ${ }^{11}$

\section{Review of published literature relating to transition of care in CF}

The majority of the published literature describes models of transition in CF based on patient readiness, and typically consider age, knowledge, and skills as the primary factors that should shape transition programs. ${ }^{10}$ Interestingly, a 2008 survey of CF centers suggested a wide range of ages for transition from 14 to 30 years with a mean of 19 years. ${ }^{12}$ Despite the lack of an evidence-based, standardized transition program for $\mathrm{CF}$ patients and the apparent heterogeneity of current practices, numerous opinion papers have been written describing the experience of individual centers or compiling survey data from multiple centers. Some of these papers expanded on the concept of age-based transition to encompass sensitivity to developmental issues that arise in adolescence such as sexuality and autonomy. ${ }^{13,14}$ A survey at one center confirmed the prevailing opinion that a majority of patients felt that a formal transition process would be useful. ${ }^{15}$ This same survey highlighted a dramatic difference in parent and adolescent patient perception with regard to the need for more disease-specific information prior to transition, with $80 \%$ of parents seeing a need, but only $38 \%$ of adolescents seeing a need. Another single-center survey administered before and after transition demonstrated that uncertainties surrounding the presumed quality of care at the adult CF care center correlated with the patients' attitude toward transition, with greater uncertainty associated with more negative attitudes. ${ }^{16}$ Similar to the divide between patients and their parents, providers perceived that patients' concerns were greater than those actually reported. ${ }^{17}$

In 2001, Flume et al published a survey of CF program directors about the current state of transition programs in US CF centers. At that time, there was consensus agreement that each $\mathrm{CF}$ center have a transition program. ${ }^{18}$ Despite this recommendation, the authors found that a minority of centers had adult CF centers approved by the Cystic Fibrosis Foundation to which patients could be transitioned and that criteria for transitioning patients were lacking. ${ }^{18}$
Simultaneously, Boyle et al published the results of a survey demonstrating that this lack of uniformity likely contributed to patient and parental concern regarding transition. ${ }^{19}$ Their results also suggested that allowing the patient to interact with the adult $\mathrm{CF}$ care team prior to transition reduced the level of concern. Others found similar results around the same time. ${ }^{20}$

There have been a handful of prior studies that have assessed concerns of patients with $\mathrm{CF}$ and their parents regarding the transition to adult care. Patient concerns include the adult practitioner's knowledge base regarding $\mathrm{CF}$, infection control in the adult health care setting, decrease in quality of care, timing of the transition (eg, when other transitions are occurring, such as going to college), and hearing difficult information from the adult practitioner which was typically shielded from them by parents. ${ }^{19,21}$ Although these studies are limited by their retrospective design and, in some cases, inclusion of participants from multiple chronic illness groups, they shed light on aspects of the transition process that could be the focus of transition interventions/ programs. For example, the study by Boyle et al suggests that introduction of the patient to the adult care team prior to transfer of care results in lower levels of concern in the patient and parents regarding leaving the pediatric $\mathrm{CF}$ center. ${ }^{19}$ A more recent study by Chaudhry et al suggested that patients who went through a transition program at their $\mathrm{CF}$ center had higher satisfaction with both the pediatric and adult $\mathrm{CF}$ care centers. ${ }^{22}$

In 2003, Patton et al made an attempt to formalize the process of transition by establishing a tool designed to gauge readiness, called the Self-Care Independence Scale. ${ }^{23}$ The tool consists of a 44-item questionnaire that is completed by the patient's parents. Data from the questionnaire were correlated with a number of other elements, including information garnered through patient interviews, and the authors reported that the tool had good internal consistency and test-retest reliability. The validity of the measure was supported by associations with the child's age, CF knowledge, and independence with non-CF self-care activities. Nonetheless, a survey of 170 of 195 US CF centers published in 2008 revealed that only half of these centers perform a transition readiness assessment of any sort. ${ }^{12}$

In sum, it appears that there are major weaknesses of the "patient readiness" approach to transition. First, it is narrowly focused on one or a few patient characteristics, such as chronological age, that may not reflect true readiness for transition. Second, these narrowly focused approaches ignore a broad array of developmental, psychosocial, and contextual 
variables that are likely to impact transition. Together, these observations highlight the need for a better core methodology for understanding, assessing, and implementing transition programs. The first step toward satisfying this goal is to identify and apply a conceptual framework for the transition process.

\section{Models of adjustment to pediatric chronic illness}

In 2006, a group of clinical scientists and health care providers from The Children's Hospital of Philadelphia and the University of Pennsylvania described two models for evaluating the psychosocial factors related to adjustment to pediatric chronic illness based on their experience with pediatric oncology patients. ${ }^{24}$ The first of these two models is called the Pediatric Psychosocial Preventative Health Model, which was established in response to data demonstrating that health care providers' assumptions about family stress in the context of life-threatening illness were not necessarily accurate. That is, most families who have a child diagnosed with cancer will function well over time despite initial and intermittent significant stress. ${ }^{24}$ To account for these data, the group established a tiered intervention model to identify those families who were most at risk for not functioning well over time and, therefore, most likely to benefit from intervention. This model is attractive because it does not assume the worst of families and aims to distribute relatively limited resources to those who need them most. Although it is difficult to draw a direct comparison between oncological diagnoses and $\mathrm{CF}$ in terms of their natural disease course, there are similarities in terms of their life-threatening nature and, anecdotally, with regard to the variability with which different families cope with the diagnosis and illness over time. In fact, this model may help to understand why some patients with CF transition from pediatric to adult $\mathrm{CF}$ care without difficulty while others struggle and suffer adverse outcomes. ${ }^{25}$

The same group of investigators proposed a second theoretical framework for understanding and intervening in children and families affected by severe illness. The National Child Traumatic Stress Network defines pediatric medical traumatic stress as "a set of psychological and physiological responses of children and their families to pain, injury, serious illness, medical procedures, and invasive or frightening treatment experiences". ${ }^{25}$ This model relies on five fundamental principles (as quoted from Kazak et al): ${ }^{25}$

- all serious medically-related events challenge beliefs about safety and invulnerability
- there is a range of normative reactions to medical traumatic events

- patients and families have a range of pre-existing psychological functioning

- a sustained focus on processes of growth and development is critical in working with children and families

- viewing the child across multiple contexts, beginning with the family and moving outward to broader systems, allows for consideration of the complex interactions that affect the well-being of children in pediatric health care. ${ }^{25}$

The model proposes that medical traumatic stress be viewed as a continuum of stress responses, both positive and negative, which occurs in a staged fashion from the time of the potentially traumatic event.

Viewed in this context, the moment that one learns of the diagnosis of CF is a potentially traumatic event. This moment is relatively easily defined for the parents of the child who is diagnosed by newborn screening. It is less clearly defined, but no less important, for the child who through developmental progression becomes aware of his or her CF diagnosis and its implications. Independent of the moment of diagnosis, living with CF may also be experienced as traumatic and provoke post-traumatic stress symptoms in parents and/or children. Moreover, the life of a child with CF is fraught with potentially traumatic events such as procedures, hospitalizations, and changes in health status. Finally, the transition from pediatric care to adult care is a time of potential uncertainty and vulnerability, and, as such, may also be perceived as traumatic. ${ }^{25}$

This model, then, would predict that the effects of transition of care likely begin with the initial discussions around the transition process and continue in phases during and for some time after the transfer event itself. The ways in which patients and families respond to discussions about transition are likely to be influenced by the presence of posttraumatic stress symptoms and/or other current stressors. For example, one may speculate that some patients and families will enter the transition process in a place of relative stability. These families may move through the process with few interventions. Other patients and families will enter the process in a less stable situation. These patients and families may require multiple interventions in order to move through the phases of transition. Therefore, the length of the transition process and the number of interventions required to complete the process may vary widely. This model also highlights the need to view traumatic events as extending not 
only to the patient-family unit and the health care team, but also to the broader context of the patient, including school and community. ${ }^{25}$ For example, the responses of teachers, peers, and community members to the patient's CF diagnosis and related treatments may shape the way in which the patient and family manage and discuss CF around others and may also contribute to overall adjustment to the disease.

The implications of these observations for transitions of care are multiple. For example, they highlight the need for an iterative process that includes periodic re-evaluation as opposed to a simpler model of linear progression. Also, they highlight the need to select for the transfer of care a time point that is separate from other stressful events. To ameliorate distress related to transition, Schwartz et $\mathrm{al}^{10}$ recommend that the process be gradual and collaborative and accompanied by positive expectations about transition outcomes.

\section{Social-ecological model of AYA readiness for transition}

The two preceding models of pediatric chronic illness are integral components of a model of transition readiness called SMART, which stands for social-ecological model of AYA readiness for transition, where "AYA" refers to adolescents and young adults. ${ }^{10}$ The authors point out that such a model is needed because multiple outcome domains may be positively or negatively affected by the mode of transition. ${ }^{10}$ In addition, the SMART model attempts to expand the conceptualization of transition beyond patient age, knowledge, and skills.

The SMART model incorporates an operational framework that includes phases of care and the discrete transfer of care. The phases of care include pediatric-based care and adult-oriented care. These are connected by a period of time in which transition readiness is assessed on multiple occasions. There are 11 components of transition readiness, comprising pre-existing factors that are unlikely or difficult to change (eg, socioeconomic status, cultural influences, disease history) and social-ecological factors related to patients, parents, and providers that may be more amenable to intervention (eg, self-efficacy, expectations, relationships). The goal of the model is not to provide a single tool per se that can identify when a patient is ready for transfer of care. Rather, there is an iterative process that involves evaluation of the multiple factors that may facilitate or hinder successful transition. ${ }^{10}$ In this fashion, the patient, family, and care team can move fluidly through the various phases of the transition process until a final decision is made on a date for transfer of care.

\section{Knowledge gaps and opportunities for research}

As reviewed above, there are subjective and objective data regarding perceptions of transition of $\mathrm{CF}$ patients from pediatric-oriented care to adult-oriented care. Nonetheless, gaps in our knowledge exist around important topics. For example, there are few data in CF that address how existing transition programs affect patient-oriented outcomes beyond satisfaction and anxiety. Further, there is a lack of data about how current transition programs in CF care centers affect objective outcomes such as forced expiratory volume in one second and frequency of pulmonary exacerbations. These knowledge gaps infer that we still do not know whether a standardized, protocol-driven transition process that incorporates individual preference and need is superior to a process that varies from center to center and from patient to patient.

In order to reasonably assess the impact of the transition process and its individual components, one must establish common transition guidelines. In CF this could be undertaken at an individual center or a small group of centers with a substantial population of adolescent patients. Although there are data to suggest that formal transition processes are beneficial in other disease groups, there remains equipoise on the benefits of a formal transition process in CF. Therefore, there is an opportunity for patients to be studied in a prospective fashion using both physiological and patient-oriented outcome measures.

To accomplish this type of study, one would need to first establish best practice guidelines based on available data. The CF care community is an ideal environment in which to establish such guidelines, as the Cystic Fibrosis Foundation has taken a leading role in establishing and communicating practice guidelines on all aspects of care (www.cff.org). Care centers are staffed with multidisciplinary providers in proportion to patient numbers so that adequate support for such a program is likely to exist at each center. In addition, the Therapeutics Development Network is well positioned to execute a study that includes both pediatric and adult CF centers. While transition is not a "therapeutic" per se, the transition event marks a discrete point in time that can be considered a medical intervention for the sake of study design.

\section{Conclusion}

The rapid advances being made in survival of CF patients make transition of care from pediatric-based centers to 
adult-based centers necessary in the majority of cases. Although some research has been conducted with respect to the perceptions of patients, families, and providers, little is known about the best method for transitioning CF patients or whether a formal transition process will result in better outcomes. Additional research is needed that takes into account the broad array of social-ecological variables that impact transition, assesses the perspectives of multiple stakeholders (patient, parents, and pediatric and adult health care professionals), considers transition as a developmental process that begins when children first begin to take on more self-care responsibilities, and incorporates prospective designs to examine the ways in which key processes and outcomes unfold over time.

\section{Disclosure}

The authors have no conflicts of interest related to the publication of this work.

\section{References}

1. Rosen DS, Blum RW, Britto M, Sawyer SM, Siegel DM; Society for Adolescent Medicine. Transition to adult health care for adolescents and young adults with chronic conditions: position paper of the Society for Adolescent Medicine. J Adolesc Health. 2003;33(4):309-311.

2. Thomas J, Cook DJ, Brooks D. Chest physical therapy management of patients with cystic fibrosis. A meta-analysis. Am J Respir Crit Care Med. 1995;151(3 Pt 1):846-850.

3. Ramsey BW, Davies J, McElvaney NG, et al. A CFTR potentiator in patients with cystic fibrosis and the G551D mutation. $N$ Engl $J$ Med. 2011;365(18):1663-1672.

4. Sawicki GS, Sellers DE, Robinson WM. High treatment burden in adults with cystic fibrosis: challenges to disease self-management. J Cyst Fibros. 2009;8(2):91-96.

5. Sawicki GS, Ren CL, Konstan MW, et al. Treatment complexity in cystic fibrosis: trends over time and associations with site-specific outcomes. J Cyst Fibros. 2013;12(5):461-467.

6. Gudas LJ, Koocher GP, Wypij D. Perceptions of medical compliance in children and adolescents with cystic fibrosis. J Dev Behav Pediatr. 1991;12(4):236-242.

7. Ricker JH, Delamater AM, Hsu J. Correlates of regimen adherence in cystic fibrosis. J Clin Psychol Med Settings. 1998;5:159-172.

8. Fuligni AJ, Eccles JS. Perceived parent-child relationships and early adolescents' orientation toward peers. Dev Psychol. 1993;29(4):622-632.

9. Coyne JC, Wortman CB, Lehman DR. The other side of support: emotional overinvolvement and miscarried helping. In: Gottieb BH, editor. Marshalling Social Support: Formats, Processes, and Effects. Newbury Park, NY: Sage; 1998.
10. Schwartz LA, Tuchman LK, Hobbie WL, Ginsberg JP. A socialecological model of readiness for transition to adult-oriented care for adolescents and young adults with chronic health conditions. Child Care Health Dev. 2011;37(6):883-895.

11. Bronfenbrenner U, Ceci SJ. Nature-nurture reconceptualized in developmental perspective: a bioecological model. Psychol Rev. 1994;101(4):568-586.

12. McLaughlin SE, Diener-West M, Indurkhya A, Rubin H, Heckmann R, Boyle MP. Improving transition from pediatric to adult cystic fibrosis care: lessons from a national survey of current practices. Pediatrics. 2008;121(5):e1160-e1166.

13. Conway SP. Transition from paediatric to adult-orientated care for adolescents with cystic fibrosis. Disabil Rehabil. 1998;20(6-7): 209-216.

14. Landau LI. Cystic fibrosis: transition from paediatric to adult physician's care. Thorax. 1995;50(10):1031-1032.

15. Westwood A, Henley L, Willcox P. Transition from paediatric to adult care for persons with cystic fibrosis: patient and parent perspectives. J Paediatr Child Health. 1999;35(5):442-445.

16. Steinkamp G, Ullrich G, Müller C, Fabel H, von der Hardt H. Transition of adult patients with cystic fibrosis from paediatric to adult care - the patients' perspective before and after start-up of an adult clinic. Eur $J$ Med Res. 2001;6(2):85-92.

17. Flume PA, Taylor LA, Anderson DL, Gray S, Turner D. Transition programs in cystic fibrosis centers: perceptions of team members. Pediatr Pulmonol. 2004;37(1):4-7.

18. Flume PA, Anderson DL, Hardy KK, Gray S. Transition programs in cystic fibrosis centers: perceptions of pediatric and adult program directors. Pediatr Pulmonol. 2001;31(6):443-450.

19. Boyle MP, Farukhi Z, Nosky ML. Strategies for improving transition to adult cystic fibrosis care, based on patient and parent views. Pediatr Pulmonol. 2001;32(6):428-436.

20. Madge S, Bryon M. A model for transition from pediatric to adult care in cystic fibrosis. J Pediatr Nurs. 2002;17(4):283-288.

21. Tuchman LK, Slap GB, Britto MT. Transition to adult care: experiences and expectations of adolescents with a chronic illness. Child Care Health Dev. 2008;34(5):557-563.

22. Chaudhry SR, Keaton M, Nasr SZ. Evaluation of a cystic fibrosis transition program from pediatric to adult care. Pediatr Pulmonol. 2013;48(7):658-665.

23. Patton SR, Graham JL, Varlotta L, Holsclaw D Jr. Measuring self-care independence in children with cystic fibrosis: the SelfCare Independence Scale (SCIS). Pediatr Pulmonol. 2003;36(2): 123-130.

24. Kazak AE, Rourke MT, Alderfer MA, Pai A, Reilly AF, Meadows AT. Evidence-based assessment, intervention and psychosocial care in pediatric oncology: a blueprint for comprehensive services across treatment. J Pediatr Psychol. 2007;32(9):1099-1110.

25. Kazak AE, Kassam-Adams N, Schneider S, Zelikovsky N, Alderfer MA, Rourke M. An integrative model of pediatric medical traumatic stress. J Pediatr Psychol. 2006;31(4):343-355.

26. Kazak AE, Cant MC, Jensen MM, et al. Identifying psychosocial risk indicative of subsequent resource use in families of newly diagnosed pediatric oncology patients. J Clin Oncol. 2003;21(17): $3220-3225$.
Patient Preference and Adherence

\section{Publish your work in this journal}

Patient Preference and Adherence is an international, peer-reviewed, open access journal focusing on the growing importance of patient preference and adherence throughout the therapeutic continuum. Patient satisfaction, acceptability, quality of life, compliance, persistence and their role in developing new therapeutic modalities and compounds to

\section{Dovepress}

optimize clinical outcomes for existing disease states are major areas of interest. This journal has been accepted for indexing on PubMed Central. The manuscript management system is completely online and includes a very quick and fair peer-review system. Visit http://www.dovepress.com/ testimonials.php to read real quotes from published authors. 